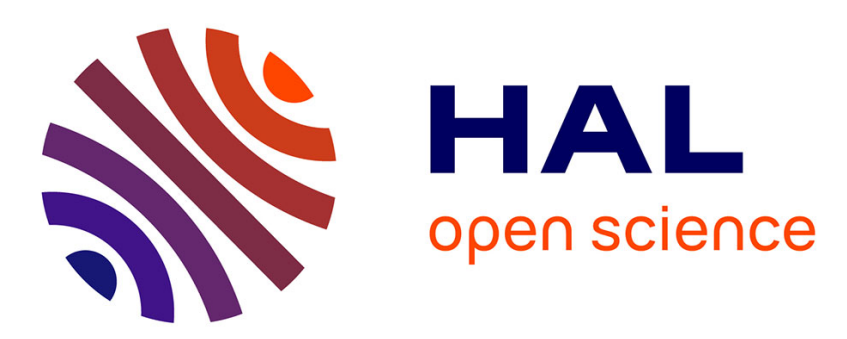

\title{
Event-Triggered Control for Systems with State Delays Using a Positive Systems Approach
}

Frederic Mazenc, Michael Malisoff, Corina Barbalata, Zhong-Ping Jiang

\section{To cite this version:}

Frederic Mazenc, Michael Malisoff, Corina Barbalata, Zhong-Ping Jiang. Event-Triggered Control for Systems with State Delays Using a Positive Systems Approach. CDC2021 - Conference on Decision and Control, Dec 2021, Austin, United States. hal-03431469

\section{HAL Id: hal-03431469 \\ https://hal.inria.fr/hal-03431469}

Submitted on 16 Nov 2021

HAL is a multi-disciplinary open access archive for the deposit and dissemination of scientific research documents, whether they are published or not. The documents may come from teaching and research institutions in France or abroad, or from public or private research centers.
L'archive ouverte pluridisciplinaire HAL, est destinée au dépôt et à la diffusion de documents scientifiques de niveau recherche, publiés ou non, émanant des établissements d'enseignement et de recherche français ou étrangers, des laboratoires publics ou privés. 


\title{
Event-Triggered Control for Systems with State Delays Using a Positive Systems Approach
}

\author{
Frédéric Mazenc, Michael Malisoff, Corina Barbalata, and Zhong-Ping Jiang
}

\begin{abstract}
We provide a new positive system approach to event-triggered feedback control of linear systems with state delays. We use an interval observer method and linear Lyapunov functions to prove global exponential stability of the closed loop systems. Our two examples illustrate the usefulness of our method for counteracting the effects of potentially destabilizing state delays.
\end{abstract}

\section{INTRODUCTION}

Event-triggered control provides the foundation for a considerable amount of current control theoretic research; see, e.g., the recent papers [6], [8], [9], [15], [18], [19], and [20]. By only changing control values when a significant event occurs, event-triggered controls can reduce the computational burden associated with control implementations, and therefore broaden the range of applications that are amenable to feedback controls. The significant events can be modeled as times when the system's state enters a given region. This is unlike the usual zero-order hold methods, where the control recomputation times do not depend on the state. One notable reformulation of much of the event-triggered literature can be done by viewing the event-triggered closed loop system as an interconnection that is amenable to smallgain methods; see, e.g., [7]. Small gain methods are useful for ensuring robustness with respect to uncertainty, but they can lead to unnecessarily frequent control recalculations and therefore also conservative results.

While recently developed computing methods can aid in the recalculation of control values, the widespread use of shared wired (or shared wireless) networked systems is strong motivation for taking communication, computation, and energy constraints into consideration [6]. This motivated event-triggered control designs, e.g.,

Key Words: stabilization, event-triggered. Supported by NSF Grants 2009659 (Malisoff and Barbalata) and 2009644 (Jiang).

F. Mazenc is with Inria Saclay, L2S-CNRS-CentraleSupélec, 3 rue Joliot Curie, 91192, Gif-sur-Yvette, France (e-mail: frederic.mazenc@12s.centralesupelec.fr)

M. Malisoff is with Department of Mathematics, Louisiana State University, Baton Rouge, LA 70803, USA (e-mail: malisoff@1su.edu)

C. Barbalata is with Department of Mechanical and Industrial Engineering, Louisiana State University, Baton Rouge, LA 70803, USA (email: cbarbalata@1su.edu)

Z.-P. Jiang is with Department of Electrical and Computer Engineering, Tandon School of Engineering, New York University, Brooklyn, NY 11201, USA (email: zjiang@nyu.edu) in [1], [2], [19], and [21]. During the same period, significant research has been devoted to positive systems, which are systems for which the nonnegative orthant is positively invariant. This led to new control analysis and designs that address some of the challenges of applying classical Lyapunov methods. Some papers on positive systems use interval observers (as defined in [4], [12], and [17]), which compute intervals that contain values of unknown states when the inequalities are viewed componentwise; see [12] and [13]. Interval observers and positive systems have enabled advances in aerospace engineering, mathematical biology, and other fields.

This motivates this paper, which provides a new positive systems based event-triggered control method yielding less conservative triggering conditions than previous methods. Building on the undelayed case in our work [14], we consider event-triggered systems with state delays that were not covered by our previous works. By proving global exponential stability using interval observers, our work is reminiscent of works like [13] that used linear Lyapunov functions. However, [13] does not use event-triggering, and we believe that this paper is the first to systematically use interval observers and positive systems to design event-triggered controls for systems with state delays.

We provide our notation, preliminaries, and class of systems in Section III. Then, in Section III. we state and prove our stabilization theorem, which can be viewed as a robustness result with respect to state delayed terms. It exhibits how smaller coefficient matrices of the state delayed term make it possible to allow longer state delays. In Section IV] we illustrate our method in two examples, where our method counteracts the effects of potentially destabilizing state delays and ensures less frequent control recalculations compared with earlier results. We close in Section $\mathrm{V}$ with summaries of our findings and suggestions for follow up research.

\section{PREliminaries}

We next review our notation, in which the dimensions of our Euclidean spaces are arbitrary unless we indicate otherwise. The arguments of our functions are omitted when no confusion can arise given the context. We set $\mathbb{Z}_{0}=\{0,1,2, \ldots\}$ and $\mathbb{N}=\mathbb{Z}_{0} \backslash\{0\}$. Given a matrix $G=$ 
$\left[g_{i j}\right] \in \mathbb{R}^{r \times s}$, we set $|G|=\left[\left|g_{i j}\right|\right]$, so the entries of $|G|$ are the absolute values of $G^{\prime}$ 's corresponding entries $g_{i j}$. In the same way, $G^{+}=\left[\max \left\{g_{i j}, 0\right\}\right], G^{-}=G^{+}-G$, and $|G|_{J}=\left[m_{i j}\right]$ where $m_{i j}=\sup _{\ell \in J}\left|g_{i j}(\ell)\right|$ when $G$ is bounded and time-varying with $J$ being an interval in $G$ 's domain. We call a square matrix Metzler provided its off-diagonal entries are all nonnegative. Given two matrices $D=\left[d_{i j}\right]$ and $E=\left[e_{i j}\right]$ having the same size, the notation $D<E$ (resp., $D \leq E$ ) means $d_{i j}<e_{i j}$ (resp., $d_{i j} \leq e_{i j}$ ) for all $i$ and $j$. Also, $D \nless E$ means that $d_{i j}>e_{i j}$ for some pair $(i, j)$. We use analogous notation for vectors.

We call a matrix $S$ positive provided $0<S$, where 0 denotes the zero matrix. For a matrix $M$ in $\mathbb{R}^{n \times n}$, we let $D_{M}$ denote the diagonal matrix such that all the diagonal entries of $M-D_{M}$ are equal to zero. We let $R_{M}=D_{M}+\left(M-D_{M}\right)^{+}$and $N_{M}=\left(M-D_{M}\right)^{-}$. Hence, in the special case where $M$ is Metzler, we have $M=R_{M}+N_{M}$. We use $I_{n}$ to denote the identity matrix in any dimension $n$. We use $C_{\text {in }}$ to denote the continuous initial functions for our delay systems, which will be defined on $[-\bar{\tau}-\nu, 0]$ for a known bound $\bar{\tau}$ on our unknown state delays $\tau$ and a constant $\nu>0$ that we specify below in Assumption 1 .

We consider systems of the form

$$
\dot{x}(t)=A_{1} x(t)+A_{2} x(t-\tau)+B u(t)
$$

with an unknown constant state delay $\tau \geq 0$ and known constant matrices $A_{i} \in \mathbb{R}^{n \times n}$ for $i=1$ and 2 and $B \in$ $\mathbb{R}^{n \times m}$, under the following assumption:

Assumption 1: There is a known bound $\bar{\tau}$ on the delay $\tau$. Also, there are known constants $p>0$ and $\nu>0$ and matrices $\Gamma>0, V>0$, and $K$ such that with the choices $A_{s}=A_{1}+A_{2}$, and with

$$
\Omega(r)=e^{A_{s} r}+\int_{0}^{r} e^{A_{s} \ell} \mathrm{d} \ell B K
$$

and

$$
\begin{aligned}
\zeta(r)= & \left|A_{2}-\Omega(r)^{-1} \int_{0}^{r} e^{A_{s} \ell} \mathrm{d} \ell B K A_{2}\right| \\
& +\int_{0}^{r}\left|\Omega(r)^{-1} e^{A_{s} \ell} A_{s} A_{2}\right| \mathrm{d} \ell \\
& +\left|\Omega(r)^{-1} A_{2}\right|
\end{aligned}
$$

and $H=A_{1}+A_{2}+B K$, the following two conditions are satisfied: (i) The inequality

$$
\left|I-\Omega(r)^{-1}\right| \leq \Gamma
$$

holds for all $r \in[0, \nu]$ and (ii) the inequalities

$$
V^{\top}\left(R_{H}+N_{H}+|B K| \Gamma\right) \leq-p V^{\top}
$$

and

$$
\begin{aligned}
& (2 \bar{\tau}+\nu) V^{\top}\left(\left|A_{2} H\right|+\left|A_{2}^{2}\right|+\left|A_{2}\right||B K| \Gamma\right. \\
& \left.+\left[I_{n}+\bar{\tau}\left|A_{2}\right|\right]|B K||\zeta|_{[0, \nu]}\right)<p V^{\top}
\end{aligned}
$$

are satisfied.
Note that since $\Omega(0)=I_{n}, \Omega(\ell)$ will be invertible for all $\ell \in[0, \nu]$ and condition (4) will hold if $\nu>0$ is small enough. Then Assumption 11 is satisfied if $A_{s}+B K$ is Hurwitz and Metzler (in which case $R_{H}+N_{H}=$ $\left.R_{H}=A_{s}+B K\right)$ and $\Gamma>0, \bar{\tau}>0, \nu>0$ are small enough; see [5, Lemma 2.3, p.41] for the existence of the required vector $V$ and constant $p>0$ in this case.

Also, if $\left(A_{s}, B\right)$ is any controllable pair, then we can select $K$ such that such that $A_{s}+B K$ has distinct negative real values as its eigenvalues. Then, after a change of coordinates (by applying a similarity transformation to $A_{s}+B K$ ), we can assume that $H$ is again Hurwitz and Metzler, so Assumption 1 then again applies when $\Gamma>0, \bar{\tau}>0$ and $\nu>0$ are small enough. In a similar way, if $\left(A_{1}, B\right)$ is controllable, then if we choose $K$ such that $A_{1}+B K$ is Hurwitz and Metzler (possibly after a change of coordinates as in the preceding case), then for any constant $\bar{\tau}>0$, we can use [5, Lemma 2.3, p.41] as before to find $p, V$, and small enough $A_{2}$ and $\nu$ such that Assumption 1 is satisfied.

\section{MAIN RESUlT}

\section{A. Statement of Theorem}

In terms of the function (3) and the function $\rho: C_{\text {in }} \rightarrow$ $\mathbb{R}^{n}$ that is defined by

$$
\rho(\phi)=\Gamma|\phi(0)|+|\zeta|_{[0, \nu]} \int_{-\bar{\tau}-\nu}^{0}|\phi(s)| \mathrm{d} s,
$$

our main result is:

Theorem 1: Consider the system (1) under Assumption 11. where $\Gamma>0, \nu, \tau$, and $K$ satisfy the requirements from Assumption 11. Consider the sequence of nonnegative numbers $t_{i}$ defined by $t_{0}=0$,

$$
\begin{aligned}
& \dot{x}(t)=A_{1} x(t)+A_{2} x(t-\tau)+B K x\left(t_{i}\right) \\
& \quad \text { if } t \in\left[t_{i}, t_{i+1}\right), \\
& \left|x(t)-x\left(t_{i}\right)\right| \leq \rho\left(x_{t}\right) \text { if } t \in\left[t_{i}, t_{i+1}\right), \text { and }
\end{aligned}
$$

For each constant $\epsilon>0$ and each $i \in \mathbb{Z}_{0}$ such that $t_{i+1}<+\infty$, there is a $t_{\star} \in\left(t_{i+1}, t_{i+1}+\epsilon\right)$ such that $\left|x\left(t_{\star}\right)-x\left(t_{i}\right)\right| \nless \rho\left(x_{t_{*}}\right)$

for all $i \in \mathbb{Z}_{0}$. Then (8)-(10) has the origin as a exponentially stable equilibrium point on $\mathbb{R}^{n}$.

Remark 1: Analogously to the cases in [14] (which did not allow state delays), solutions of the closed loop event-triggered control system (8)-(10) are defined by the following recursive method. Starting from any initial state $x(0)$, we solve the differential equations in $(8)$ on the maximum interval $[0, \bar{v})$ on which the inequality in (9) continues to hold. If $\bar{v}=+\infty$, then the control value is kept at $K x(0)$ and $t_{1}=+\infty$. If the inequality in 9 ceases to hold at some finite time, then choose $t_{1}$ to be the infimum of such times when (9) fails to hold (and the first part of our proof of Theorem 1 will give 
$t_{1}-t_{0} \geq \nu$, where $\nu$ is from Assumption 11, in which case the condition from 100 holds for $i=0$. Then solve the system of differential equations in (8) with $i=1$ on $\left[t_{1}, t_{2}\right)$, using the initial state $x\left(t_{1}\right)$ obtained in the preceding argument, and replace $t_{0}=0$ and $t_{1}$ in the preceding argument by $t_{1}$ and $t_{2}$, respectively. Arguing inductively then defines the solutions of (8)-(10) for all $t \geq 0$, which all satisfy the exponential stability estimate from the conclusion of the theorem.

Remark 2: A key feature of our approach is our method for computing our lower bound $\nu$ on the intersample times $t_{i+1}-t_{i}$ that is independent of the initial state. This is made possible by our novel positive systems and interval observer approaches, and it contrasts with [3], where the lower bound on the inter-sample times depended on the initial state.

\section{B. Proof of Theorem 1}

The proof is organized into three parts. In the first part, we show that the Zeno phenomenon does not occur. In the second part, we show how the conclusion of the theorem would follow if the dynamics for

$$
x_{*}=\bar{x}-\underline{x}
$$

are globally exponentially stable to 0 on $\mathbb{R}^{n}$, where $(\bar{x}, \underline{x})$ is the vector of states of an interval observer for the state $x$. In the last part, we show the required exponential stability property for the $x_{*}$ dynamics.

First Part. We prove that $t_{i+1} \geq t_{i}+\nu$ for all $i \in \mathbb{Z}_{\geq 0}$ and for all solutions of $(8)-(10)$, which will prove that the Zeno phenomenon does not occur. Let

$$
z(t)=x(t)+A_{2} \int_{t-\tau}^{t} x(m) \mathrm{d} m .
$$

Then for all solutions $x(t)$ of $(8)$ for all initial states, and for all $t \in\left[t_{i}, t_{i}+\nu\right]$, we have

$$
\begin{aligned}
\dot{z}(t)= & A_{s} x(t)+B K x\left(t_{i}\right) \\
= & A_{s}\left[z(t)-A_{2} \int_{t-\tau}^{t} x(m) \mathrm{d} m\right] \\
& +B K\left[z\left(t_{i}\right)-A_{2} \int_{t_{i}-\tau}^{t_{i}} x(m) \mathrm{d} m\right] .
\end{aligned}
$$

Here and in the sequel, all equalities and inequalities should be understood to hold along all solutions of the closed loop system from the theorem and all $i \in \mathbb{Z}_{\geq 0}$, unless otherwise indicated. Grouping the terms gives

$$
\begin{aligned}
& \dot{z}(t)=A_{s} z(t)+B K z\left(t_{i}\right) \\
& -A_{s} A_{2} \int_{t-\tau}^{t} x(m) \mathrm{d} m-B K A_{2} \int_{t_{i}-\tau}^{t_{i}} x(m) \mathrm{d} m
\end{aligned}
$$

for all $t \in\left[t_{i}, t_{i}+\nu\right]$. By applying the method of variation of parameters to (14), we get

$$
\begin{aligned}
z(t)= & e^{A_{s}\left(t-t_{i}\right)} z\left(t_{i}\right)+\int_{t_{i}}^{t} e^{A_{s}(t-\ell)} \mathrm{d} \ell B K z\left(t_{i}\right) \\
& +\int_{t_{i}}^{t} e^{A_{s}(t-\ell)}\left[-A_{s} A_{2} \Delta(\ell)\right. \\
& \left.-B K A_{2} \Delta\left(t_{i}\right)\right] \mathrm{d} \ell \\
= & \Omega\left(t-t_{i}\right) z\left(t_{i}\right)+\int_{t_{i}}^{t} e^{A_{s}(t-\ell)}\left[-A_{s} A_{2} \Delta(\ell)\right. \\
& \left.-B K A_{2} \Delta\left(t_{i}\right)\right] \mathrm{d} \ell
\end{aligned}
$$

where

$$
\Delta(\ell)=\int_{\ell-\tau}^{\ell} x(m) \mathrm{d} m
$$

Using the definition of $z$, we deduce that

$$
\begin{aligned}
& x(t)+A_{2} \Delta(t)=\Omega\left(t-t_{i}\right)\left[x\left(t_{i}\right)+A_{2} \Delta\left(t_{i}\right)\right] \\
& +\int_{t_{i}}^{t} e^{A_{s}(t-\ell)}\left[-A_{s} A_{2} \Delta(\ell)-B K A_{2} \Delta\left(t_{i}\right)\right] \mathrm{d} \ell .
\end{aligned}
$$

Therefore, for all $t \in\left[t_{i}, t_{i}+\nu\right]$, we have

$$
\begin{aligned}
& \Omega\left(t-t_{i}\right)^{-1} x(t)=-\Omega\left(t-t_{i}\right)^{-1} A_{2} \Delta(t) \\
& +x\left(t_{i}\right)+A_{2} \Delta\left(t_{i}\right) \\
& +\Omega\left(t-t_{i}\right)^{-1} \int_{t_{i}}^{t} e^{A_{s}(t-\ell)}\left[-A_{s} A_{2} \Delta(\ell)\right. \\
& \left.-B K A_{2} \Delta\left(t_{i}\right)\right] \mathrm{d} \ell .
\end{aligned}
$$

Rearranging the terms in (18), we obtain

$$
\begin{aligned}
& x\left(t_{i}\right)-x(t)=\left[\Omega\left(t-t_{i}\right)^{-1}-I\right] x(t)-A_{2} \Delta\left(t_{i}\right) \\
& +\Omega\left(t-t_{i}\right)^{-1} \int_{t_{i}}^{t} e^{A_{s}(t-\ell)}\left[A_{s} A_{2} \Delta(\ell)\right. \\
& \left.+B K A_{2} \Delta\left(t_{i}\right)\right] \mathrm{d} \ell+\Omega\left(t-t_{i}\right)^{-1} A_{2} \Delta(t)
\end{aligned}
$$

and so also

$$
\begin{aligned}
& x\left(t_{i}\right)-x(t)=\left[\Omega\left(t-t_{i}\right)^{-1}-I\right] x(t)+\left[-A_{2}\right. \\
& \left.+\Omega\left(t-t_{i}\right)^{-1} \int_{t_{i}}^{t} e^{A_{s}(t-\ell)} B K A_{2} d \ell\right] \Delta\left(t_{i}\right) \\
& +\Omega\left(t-t_{i}\right)^{-1} \int_{t_{i}}^{t} e^{A_{s}(t-\ell)} A_{s} A_{2} \Delta(\ell) \mathrm{d} \ell \\
& +\Omega\left(t-t_{i}\right)^{-1} A_{2} \Delta(t) .
\end{aligned}
$$

It follows that

$$
\begin{aligned}
& \left|x\left(t_{i}\right)-x(t)\right| \leq \Gamma|x(t)|+\mid-A_{2} \\
& +\Omega\left(t-t_{i}\right)^{-1} \int_{t_{i}}^{t} e^{A_{s}(t-\ell)} B K A_{2} d \ell|| \Delta\left(t_{i}\right) \mid \\
& +\int_{t_{i}}^{t}\left|\Omega\left(t-t_{i}\right)^{-1} e^{A_{s}(t-\ell)} A_{s} A_{2}\right||\Delta(\ell)| \mathrm{d} \ell \\
& +\left|\Omega\left(t-t_{i}\right)^{-1} A_{2}\right||\Delta(t)|
\end{aligned}
$$

and therefore also

$$
\begin{aligned}
& \left|x\left(t_{i}\right)-x(t)\right| \\
& \leq \Gamma|x(t)|+\zeta\left(t-t_{i}\right) \int_{t-\nu-\bar{\tau}}^{t}|x(m)| \mathrm{d} m .
\end{aligned}
$$

with $\zeta$ defined in (3). Hence, the inequality in (9) holds on $\left[t_{i}, t_{i}+\nu\right]$. We conclude that $t_{i+1}-t_{i} \geq \nu$, so the Zeno phenomenon does not occur.

Second Part. We show that with the choice $x_{*}=\bar{x}-$ $\underline{x}$ for a state $(\bar{x}, \underline{x})$ of an interval observer for $x$, the solutions of the closed loop system satisfy

$$
|x(t)| \leq x_{*}(t)
$$

for all $t \geq 0$. This will replace the search for a global exponential stability estimate in the state variable $x(t)$ with an easier task of finding the corresponding estimate for the dominating variable $x_{*}(t)$. 
To this end, first notice that by the first part of the proof, the solutions of the closed loop system in the statement of the theorem satisfy

$$
\begin{aligned}
\dot{x}(t)= & H x(t)+A_{2}(x(t-\tau)-x(t)) \\
& +B K\left(x\left(t_{i}\right)-x(t)\right) \\
= & H x(t)+A_{2}(x(t-\tau)-x(t))+\mu(t)
\end{aligned}
$$

for all $t \geq 0$, where $H=A_{s}+B K$ and

$$
\begin{aligned}
& |\mu(t)| \leq \\
& |B K|\left(\Gamma|x(t)|+|\zeta|_{[0, \nu]} \int_{t-\nu-\bar{\tau}}^{t}|x(m)| \mathrm{d} m\right) .
\end{aligned}
$$

We have

$$
\dot{x}(t)=H x(t)-A_{2} \int_{t-\tau}^{t} \dot{x}(m) \mathrm{d} m+\mu(t) .
$$

Thus

$$
\begin{aligned}
& \dot{x}(t)=H x(t)-A_{2} \int_{t-\tau}^{t}[H x(m) \\
& \left.+A_{2}(x(m-\tau)-x(m))+\mu(m)\right] \mathrm{d} m+\mu(t)
\end{aligned}
$$

and so also

$$
\begin{aligned}
& \dot{x}(t)=H x(t)-A_{2} H \int_{t-\tau}^{t} x(m) \mathrm{d} m \\
& -A_{2}^{2} \int_{t-2 \tau}^{t-\tau} x(m) \mathrm{d} m+A_{2}^{2} \int_{t-\tau}^{t} x(m) \mathrm{d} m+\mu^{\sharp}(t)
\end{aligned}
$$

for all $t \geq \tau$, where

$$
\mu^{\sharp}(t)=\mu(t)-A_{2} \int_{t-\tau}^{t} \mu(m) \mathrm{d} m .
$$

This leads us to study the dynamic extension

$$
\begin{aligned}
\dot{\bar{x}}(t)= & R_{H} \bar{x}(t)-N_{H} \underline{x}(t)+\left(\mu^{\sharp}(t)\right)^{+} \\
& -\left(A_{2} H\right)^{+} \int_{t-\tau}^{t} \underline{x}(m) \mathrm{d} m \\
& +\left(A_{2} H\right)^{-} \int_{t-\tau}^{t} \bar{x}(m) \mathrm{d} m \\
& -\left(A_{2}^{2}\right)^{+} \int_{t-2 \tau}^{t-\tau} \underline{x}(m) \mathrm{d} m \\
& +\left(A_{2}^{2}\right)^{-} \int_{t-2 \tau}^{t-\tau} \bar{x}(m) \mathrm{d} m \\
& +\left(A_{2}^{2}\right)^{+} \int_{t-\tau}^{t} \bar{x}(m) \mathrm{d} m \\
& -\left(A_{2}^{2}\right)^{-} \int_{t-\tau}^{t} \underline{x}(m) \mathrm{d} m \\
& R_{H} \underline{x}(t)-N_{H} \bar{x}(t)-\left(\mu^{\sharp}(t)\right)^{-} \\
& -\left(A_{2} H\right)^{+} \int_{t-\tau}^{t} \bar{x}(m) \mathrm{d} m \\
& +\left(A_{2} H\right)^{-} \int_{t-\tau}^{t} \underline{x}(m) \mathrm{d} m \\
& -\left(A_{2}^{2}\right)^{+} \int_{t-2 \tau}^{t-\tau} \bar{x}(m) \mathrm{d} m \\
& +\left(A_{2}^{2}\right)^{-} \int_{t-2 \tau}^{t-\tau} \underline{x}(m) \mathrm{d} m \\
& +\left(A_{2}^{2}\right)^{+} \int_{t-\tau}^{t} \underline{x}(m) \mathrm{d} m \\
& -\left(A_{2}^{2}\right)^{-} \int_{t-\tau}^{t} \bar{x}(m) \mathrm{d} m,
\end{aligned}
$$

where the initial functions for 30 are chosen such that

$$
\underline{x}(s) \leq x(s) \leq \bar{x}(s)
$$

for all $s \in[-2 \tau, 0]$. Then the formulas $H=R_{H}-$ $N_{H}, A_{2} H=\left(A_{2} H\right)^{+}-\left(A_{2} H\right)^{-1}$, and the analogous formula for $A_{2}^{2}$ and standard positive systems arguments (e.g., from [14]) imply that $\bar{e}(t) \geq 0 \geq \underline{e}(t)$ and therefore also $\bar{x}(t) \geq x(t) \geq \underline{x}(t)$ for all $t \geq 0$, where $\bar{e}=\bar{x}-x$ and $\underline{e}=x-\underline{x}$.

Similar reasoning gives $\bar{x}(t) \geq 0 \geq \underline{x}(t)$ for all $t \geq 0$ (by using 30 to show that $(\bar{x}(t),-\underline{x}(t))$ is a solution of a positive system and therefore is nonnegative valued) and so also $\underline{x}(t)-\bar{x}(t) \leq x(t) \leq \bar{x}(t)-\underline{x}(t)$, which gives the desired bound (23).

Third Part. We use the bound 23) to prove the global exponential stability conclusion of the theorem. To this end, first notice that $x_{*}=\bar{x}-\underline{x}$ satisfies

$$
\begin{aligned}
& \dot{x}_{*}(t)=\left|\mu^{\sharp}(t)\right| \\
& +\left(R_{H}+N_{H}\right) x_{*}(t)+\left|A_{2} H\right| \int_{t-\tau}^{t} x_{*}(m) \mathrm{d} m \\
& +\left|A_{2}^{2}\right| \int_{t-2 \tau}^{t-\tau} x_{*}(m) \mathrm{d} m+\left|A_{2}^{2}\right| \int_{t-\tau}^{t} x_{*}(m) \mathrm{d} m,
\end{aligned}
$$

because $|M|=M^{+}+M^{-}$for each matrix $M$. Moreover, we can combine (25) and (23) to obtain

$$
\begin{aligned}
& \left|\mu^{\sharp}(t)\right| \\
& \leq|B K|\left(\Gamma x_{*}(t)+|\zeta|_{[0, \nu]} \int_{t-\nu-\bar{\tau}}^{t} x_{*}(m) \mathrm{d} m\right) \\
& +\bar{\alpha} \int_{t-\tau}^{t}\left(\Gamma x_{*}(\ell)+|\zeta|_{[0, \nu]} \int_{\ell-\nu-\bar{\tau}}^{\ell} x_{*}(r) \mathrm{d} r\right) \mathrm{d} \ell
\end{aligned}
$$

for all $t \geq \nu+\tau$, where

$$
\bar{\alpha}=\left|A_{2}\right||B K| .
$$

Hence, by the comparison principle, the $x_{*}$ dynamics will be globally exponentially stable to 0 on $\mathbb{R}^{n}$ if the positive system

$$
\begin{aligned}
\dot{g}(t)= & \left(R_{H}+N_{H}+|B K| \Gamma\right) g(t) \\
& +\left|A_{2} H\right| \int_{t-\tau}^{t} g(m) \mathrm{d} m \\
& +\left|A_{2}^{2}\right| \int_{t-2 \tau}^{t-\tau} g(m) \mathrm{d} m \\
& +\left|A_{2}^{2}\right| \int_{t-\tau}^{t} g(m) \mathrm{d} m \\
& +\left|A_{2}\right| \int_{t-\tau}^{t}|B K|(\Gamma g(\ell) \\
& \left.+|\zeta|_{[0, \nu]} \int_{\ell-\nu-\tau}^{\ell} g(m) \mathrm{d} m\right) \mathrm{d} \ell \\
& +|B K||\zeta|_{[0, \nu]}^{t} \int_{t-\nu-\tau}^{t} g(m) \mathrm{d} m
\end{aligned}
$$

is globally exponentially stable to 0 on $\mathbb{R}^{n}$. Hence, it remains to prove that 35 is globally exponentially stable to 0 on $\mathbb{R}^{n}$, which will imply the conclusion of the theorem because of our bound (23) and our assumptions on the initial states for $\bar{x}$ and $\underline{x}$.

To this end, note that our condition (5) on the vector $V>0$ and $p>0$ imply that along all nonnegative valued solutions of (35), the function

$$
U(g)=V^{\top} g
$$


satisfies

$$
\begin{aligned}
\dot{U}(t) \leq & -p V^{\top} g(t)+V^{\top}\left|A_{2} H\right| \int_{t-\tau}^{t} g(m) \mathrm{d} m \\
& +V^{\top}\left|A_{2}^{2}\right| \int_{t-2 \tau}^{t-\tau} g(m) \mathrm{d} m \\
& +V^{\top}\left|A_{2}^{2}\right| \int_{t-\tau}^{t} g(m) \mathrm{d} m \\
& +V^{\top}\left|A_{2}\right| \int_{t-\tau}^{t}|B K|(\Gamma|g(\ell)| \\
& \left.+|\zeta|_{[0, \nu]} \int_{\ell-\nu-\tau}^{\ell} g(m) \mathrm{d} m\right) \mathrm{d} \ell \\
& +V^{\top}|B K||\zeta|_{[0, \nu]} \int_{t-\nu-\tau}^{t} g(m) \mathrm{d} m \\
\leq & -p V^{\top} g(t)+\left(V^{\top}\left|A_{2} H\right|\right. \\
& +V^{\top}\left|A_{2}^{2}\right|+V^{\top}|B K||\zeta|_{[0, \nu]} \\
& \left.+V^{\top} \bar{\alpha} \Gamma\right) \int_{t-2 \tau-\nu}^{t} g(m) \mathrm{d} m \\
& +V^{\top} \bar{\alpha}|\zeta|_{[0, \nu]}^{t} \int_{t-\tau}^{t} \int_{\ell-\nu-\tau}^{\ell} g(m) \mathrm{d} m \mathrm{~d} \ell \\
\leq & -p V^{\top} g(t)+V^{\top} \mathcal{H} \int_{t-2 \tau-\nu}^{t} g(m) \mathrm{d} m,
\end{aligned}
$$

where

$$
\begin{aligned}
\mathcal{H}= & \left|A_{2} H\right|+\left|A_{2}^{2}\right|+\left|A_{2}\right||B K| \Gamma \\
& +\left(I_{n}+\tau\left|A_{2}\right|\right)|B K||\zeta|_{[0, \nu]} .
\end{aligned}
$$

Here and in the sequel, equalities and inequalities should be understood to hold along all solutions of (35) for all $t \geq \nu+\tau$, unless otherwise noted. Let $\epsilon \in(0,1)$ be a constant such that $(1+\epsilon)(2 \tau+\nu) V^{\top} \mathcal{H}<p V^{\top}$; such an $\epsilon$ exists because of our assumption (6). Let

$$
\begin{aligned}
& \bar{U}\left(g_{t}\right)=U(g(t)) \\
& +(1+\epsilon) V^{\top} \mathcal{H} \int_{t-2 \tau-\nu}^{t} \int_{\ell}^{t} g(m) \mathrm{d} m \mathrm{~d} \ell .
\end{aligned}
$$

Then

$$
\begin{aligned}
\dot{\bar{U}}(t) \leq & {\left[-p V^{\top}+(1+\epsilon)(2 \tau+\nu) V^{\top} \mathcal{H}\right] g(t) } \\
& -\epsilon V^{\top} \mathcal{H} \int_{t-2 \tau-\nu}^{t} g(m) \mathrm{d} m .
\end{aligned}
$$

Since the quantity in squared brackets in (40) is negative and

$$
\int_{t-2 \tau-\nu}^{t} g(m) \mathrm{d} m \geq \frac{1}{2 \tau+\nu} \int_{t-2 \tau-\nu}^{t} \int_{\ell}^{t} g(m) \mathrm{d} m \mathrm{~d} \ell
$$

holds for all $t \geq 2 \tau+\nu$, this gives the desired exponential decay of $\bar{U}$ along all solutions of (35), which gives the stability condition for 35 to complete the proof.

\section{ILLUSTRATIONS}

a) Theoretical example: In the special case where $A_{2}$ is the zero matrix, the preceding result agrees with the main result in [14] which did not allow state delays. Therefore, it is of interest to generalize the twodimensional example from [14, Section IV] to cases where there is also a state delayed term. Hence, we consider the system

$$
\left\{\begin{aligned}
\dot{x}_{1}(t)= & x_{1}(t)+\frac{1}{2} x_{2}(t)+a x_{1}(t-\tau) \\
& +b x_{2}(t-\tau)+u \\
\dot{x}_{2}(t)= & \frac{3}{2} x_{1}(t)+c x_{1}(t-\tau) \\
& +d x_{2}(t-\tau)+u
\end{aligned}\right.
$$

where $x_{1}$ and $x_{2}$ are valued in $\mathbb{R}$, the coefficients $a, b$, $c$, and $d$ are known constants, $\tau>0$ is an unknown constants delay, and $u$ is the input, which agrees with the example from [14, Section IV] in the special case where $a=b=c=d=0$. To apply Theorem 1 we will choose the same $K$ and $\Gamma$ that were used in the $a=b=c=d=0$ case in [14], and then we determine how large a state delay $\tau$ can be allowed while still satisfying the requirements from Theorem 1 to achieve global exponential stability. For simplicity, we will choose $a=b=c=d=0.01$, but analogous reasoning applies with other choices of these constants.

Following [14], we therefore choose $K=$ $[-4 / 3,-1 / 3]$ and all entries of $\Gamma$ being $\Gamma_{i j}=0.045$. Then, with the choices $V=[1,0.9]$ and $p=0.05$, the largest values of $\nu$ and $\tau$ for which Assumption 11 is satisfied are $\nu=0.035$ and $\tau=0.1$. Since state delays were beyond the scope of [14] and other earlier works, this illustrates the value of our new Theorem 1 for quantifying the ability of our event-triggered control to compensate for state delays.

b) Marine robotics example: A second example is given for the control of the depth and pitch degrees-offreedom (or DOF) of an autonomous underwater vehicle (or AUV). The vehicle is equipped with a Doppler Velocity Logger (or DVL) that estimates the velocity of the vehicle. When working in close proximity to the sea floor, the DVL experiences bottom lock that leads to delays in the state of the vehicle. In this example, we discuss the design of the control system for the depth plane considering these delays in the heave and pitch velocity measurements.

As presented in [16, Equation (9.28)], after linearization and assuming that the vehicle is neutrally buoyant, the linearized equations of motion in the depth plane are given by

$$
\begin{aligned}
& \left(m-X_{\dot{w}(t)}\right) \dot{w}(t)-\left(m x_{g}+Z_{\dot{q}}\right) \dot{q}(t) \\
& -Z_{w} w(t-\tau)-\left(m U(t)+z_{q}\right) q(t-\tau)=Z_{\gamma_{s}} u_{Z} \\
& \text { and }\left(m x_{g}+M_{\dot{w}}(t)\right) \dot{w}(t)+\left(I_{y y}-M_{\dot{q}}\right) \dot{q}(t) \\
& -M_{w} w(t-\tau)+\left(m x_{g} U-M_{q}\right) q(t-\tau)-M_{\theta} \theta \\
& =M_{\gamma_{s}} u_{M}
\end{aligned}
$$

whose parameters were experimentally computed and presented in [16]. Its states are the depth and pitch

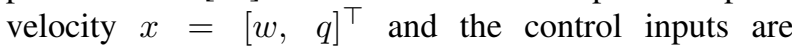
$u=\left[u_{Z}, u_{M}\right]^{\top}$ representing the force and moment required to produce the motion of the vehicle. For this simulation the surge nominal velocity was considered as $U=0.1 \mathrm{~m} / \mathrm{s}$.

For this system, we chose $K=[0.59,0.23]$, the matrix $\Gamma=\left[\Gamma_{i j}\right]$ having entries $\Gamma_{11}=0.48, \Gamma_{12}=0.06$, $\Gamma_{21}=0.06$, and $\Gamma_{22}=0.3, V=\left[\begin{array}{ll}0.11, & 0.15\end{array}\right]^{\top}$, and $p=0.1$, which satisfy our requirements from 
Assumption 1] with $\nu=0.07$ and $\tau=0.1$. With the preceding design parameters, we used MATLAB to simulate the depth plane of the model and its corresponding controller from Theorem 1. In Fig. 1, we show our MATLAB simulation results. Fig. 1 shows that the

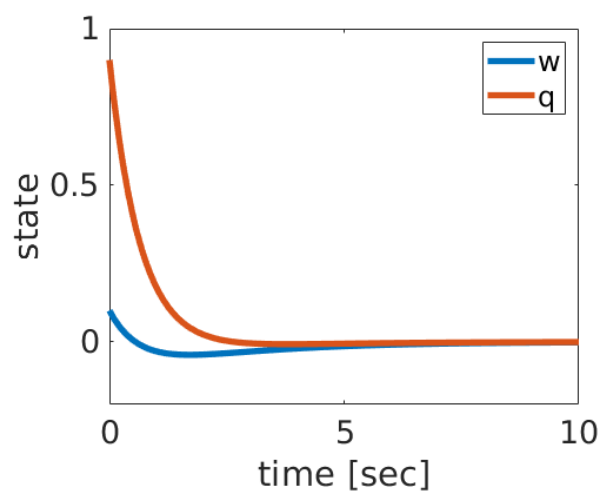

Fig. 1: Depth and pitch DOFs using Theorem 1 control depth and pitch reach steady-state in less than 4 seconds with no overshoot for pitch, and minimum overshoot for depth caused by the coupling to the pitch DOF. This illustrates our event-triggered state delay compensating feedback control in a practical example using experimentally validated values of the model parameters.

\section{Conclusions}

We provided a new event-triggered feedback control design for a class of linear systems having state delays, using positive system and interval observer approaches, which enable us to prove exponential convergence properties using linear Lyapunov functions. As illustrated in [14] in the special case where there are no state delays, our positive systems approach can provide advantages over prior methods, by obtaining larger guaranteed lower bounds on the inter-sample interval lengths $t_{i+1}-t_{i}$ and therefore less frequent control recalculations as compared with small gain or other earlier methods, and these advantages are also present in our treatment of more complicated state delayed systems in this work. Our guaranteed lower bound on the inter-sample interval lengths is independent of the initial state. We aim to extend our analysis to nonlinear systems with outputs and uncertain coefficients, using event-triggered analogs of our finite time or continuous-discrete observer designs [10], [11], as well as to event-triggered adaptive dynamic programming based optimization [22] under state delays.

\section{REFERENCES}

[1] Brunner, F., W. Heemels, and F. Allgower. Robust event-triggered MPC with guaranteed asymptotic bound and average sampling rate. IEEE Transactions on Automatic Control, 62(11):56945709, 2017.

[2] Dolk, V., and M. Heemels. Event-triggered control systems under packet losses. Automatica, 80:143-155, 2017.
[3] Durand, S., N. Marchand, and J.F.G. Castellanos. General formula for event-based stabilization of nonlinear systems with delays in the state. In Delays and Networked Control Systems, A. Seuret, L. Hetel, J. Daafouz, and K. Johansson, Eds., Advances in Delays and Dynamics Vol. 6, Springer, New York, NY, 2016, pp. $59-77$.

[4] Gouzé, J.-L., A. Rapaport, and Z. Hadj-Sadok. Interval observers for uncertain biological systems. Ecological Modelling, 133(12):45-56, 2000.

[5] Haddad, W., V. Chellaboina, and Q. Hui. Nonnegative and Compartmental Dynamical Systems. Princeton University Press, Princeton, NJ, 2010.

[6] Heemels, W., K. Johansson, and P. Tabuada. An introduction to event-triggered and self-triggered control. In Proceedings of 51st IEEE Conference on Decision and Control (Maui, Hawaii, 10-13 December 2012), pp. 3270-3285.

[7] Jiang, Z.-P., and T. Liu. Small-gain theory for stability and control of dynamical networks: A survey. Annual Reviews in Control, 46:58-79, 2018.

[8] Liu, D., and G.-H. Yang. Dynamic event-triggered control for linear time-invariant systems with $L_{2}$-gain performance. International Journal of Robust and Nonlinear Control, 29(2):507-518, 2019.

[9] Liu, T., P. Zhang, and Z.-P. Jiang. Robust Event-Triggered Control of Nonlinear Systems. Springer, New York, NY, 2020.

[10] Mazenc, F., S. Ahmed, and M. Malisoff. Finite time estimation through a continuous-discrete observer. International Journal of Robust and Nonlinear Control, 28(16):4831-4849, 2018.

[11] Mazenc, F., V. Andrieu, and M. Malisoff. Design of continuousdiscrete observers for time-varying nonlinear systems. Automatica, 57(7):135-144, 2015.

[12] Mazenc, F., and O. Bernard. Interval observers for linear timeinvariant systems with disturbances. Automatica, 47(1):140-147, 2011.

[13] Mazenc, F., and M. Malisoff. Stability analysis for time-varying systems with delay using linear Lyapunov functionals and a positive systems approach. IEEE Transactions on Automatic Control, 61(3):771-776, 2016.

[14] Mazenc, F., M. Malisoff, C. Barbalata, and Z.-P. Jiang. Eventtriggered control using a positive systems approach. In Proceedings of the European Control Conference (29 June-2 July 2021), to appear, http://www.math.lsu.edu/ malisoff/.

[15] Peralez, J., V. Andrieu, M. Nadri, and U. Serres. Event-triggered output feedback stabilization via dynamic high-gain scaling. IEEE Transactions on Automatic Control, 63(8):2537-2549, 2018.

[16] Prestero, T. Verification of a Six-Degree of Freedom Simulation Model for the REMUS Autonomous Underwater Vehicle. MS Thesis, Massachusetts Institute of Technology, Cambridge, MA, 2001.

[17] Raissi, T., D. Efimov, and A. Zolghadri. Interval state estimation for a class of nonlinear systems. IEEE Transactions on Automatic Control, 57(1):260-265, 2012.

[18] Selivanov, A., and E. Fridman. Event-triggered $\mathcal{H}_{\infty}$ control: a switching approach. IEEE Transactions on Automatic Control, 61(10):3221-3226, 2016

[19] Tabuada, P. Event-triggered real-time scheduling of stabilizing control tasks. IEEE Transactions on Automatic Control, 52(9):1680-1685, 2007.

[20] Wang, X., and M. Lemmon. Self-triggered feedback control systems with finite-gain $L_{2}$ stability. IEEE Transactions on Automatic Control, 54(3):452-467, 2009.

[21] Yook, J., D. Tilbury, and N. Soparkar. Trading computation for bandwidth: Reducing communication in distributed control systems using state estimators. IEEE Transactions on Control Systems Technology, 10(4):503-518, 2002.

[22] Zhao, F., W. Gao, Z.-P. Jiang, and T. Liu. Event-triggered adaptive optimal control with output feedback: an adaptive dynamic programming approach. IEEE Transactions on Neural Networks and Learning Systems, to appear, https://doi.org/10.1109/TNNLS.2020.3027301. 